\title{
IMPROVING QUALITY OF SERVICE IN IP NETWORKS FOR MULTIMEDIA APPLICATIONS WITH OPTIMAL FRAGMENTATION
}

\author{
K. Sasi Kala Rani and J. Suganthi \\ Faculty of Computer Science and Engineering, \\ Hindusthan College of Engineering and Technology, Othakalmandapam, Coimbatore-641 032, India
}

Received 2012-10-09; Received 2013-01-07; Accepted 2014-03-03

\begin{abstract}
The tremendous increase of Internet users and the wide ranging need to support both multimedia traffic and other traffic leads to service differentiation schemes. Multimedia applications have uncompromising and strict deadlines and the packets are worthless if they arrive late than their associated deadlines. The aim of the proposed system is to improve the quality of service by increasing the number of multimedia packets that reaches the destination before their associated deadline without affecting the fairness of other data. The proposed algorithm estimates the optimal packet size for transmission and puts forward a Service Differentiation scheme that drops the packets based on the size and deadline of the packets. Packet loss percentage is observed to be reduced in the proposed system.
\end{abstract}

Keywords: Optimal Packet Size, Packet Loss, Deadline, SDP, Modified RED

\section{INTRODUCTION}

The magnificent increase of Internet users has flooded the Internet with diverse applications. The applications contribute heterogeneous data which comprise of real time data like voice, video and traditional data like e-mail, file transfer to the internet. The internet traffic has different QoS requirements. Qos parameters include data rate, delay, jitter, ordered delivery, loss, error rates (Steinmetz and Nahrstedt, 2004). Service principle called Less Impact Better Service (LIBS) (Mamatas and Tsaoussidis, 2010) leads to a novel service differentiation method, namely Sizeoriented Dropping Policy (SDP), which uses packet size to categorize time-sensitive from delay-tolerant flows and prioritize packet dropping probability, accordingly. A significant increase on the perceived quality of real-time applications was perceived.

For example the real time data flows are intolerant to delay, packet loss and jitter. Traditional data flows are intolerant to packet loss but tolerant to delay and jitter. The flows are aggregated in diffserv model where packets are classified as belonging to a flow if they have the same marking in Type of Service (ToS) byte in the IP header. Scheduling disciplines give priority to QoS sensitive flows in routers but also maintains the fairness of non-priority queue (Bourenene et al., 2008).

More packet loss in the case of multimedia application degrades the quality of the video and voice at the receiving end.

The proposed system focuses on improving the perceived quality of picture and sound by increasing the number of multimedia packets that reaches the destination before expiry of their deadline. Multimedia data are differentiated by the size. Small packets usually correspond to the real time applications like video and voice over internet protocol and bulk data applications use big packets (Dimitriou et al., 2010). The proposed system optimizes Sizeoriented Dropping Policy (SDP) by incorporating the following:

- Calculation of optimal packet size of multimedia data thereby increasing the number of packets that reaches the destination on time

- Dropping packets based on size as well as remaining deadline Corresponding Author: K. Sasi Kala Rani, Department of Computer Science and Engineering, Hindusthan College of Engineering and
Technology, Othakalmandapam, Coimbatore-641 032, India 
The study is organized as follows:

- Section 2 discusses the related work

- Section 3 explains the proposed system

- Section 4 compares the packet loss percentage of SDP and proposed system

\section{RELATED WORK}

Multimedia applications such as video streaming, which are delay sensitive and bandwidth sensitive are growing rapidly over internet. Video packets can tolerate some packet loss. Lost video packets induce different levels of quality degradation.

Service differentiation has been developed on the basis of resource allocation with the corresponding service requirements of diverse applications. Applications with strict delay, jitter and packet loss constraints can be satisfied by prioritizing real-time data over bulk data transfers (Georgiou et al., 2011).

Throughput of multimedia applications can be improved by fragmenting multimedia packets into optimal fragments. Fragmentation of packet calls a trade off between reducing the number of overhead bits per packet by using large fragment size and reducing transmission error rate by using small packets (Kambhatla et al., 2012). Hence multimedia data has to be differentiated from other data that can be done using packet size. The diffserv approach enables prioritization by marking with corresponding service identifiers. (Bourenene et al., 2008; Dimitriou et al., 2010)

Each packet has a deadline associated with it. If packets belonging to real time applications are delayed beyond deadline, those packets are considered as lost since those packets will be discarded at their destination (Li, 2009). Non-Congestive Queuing Plus (NCQ+) promotes applications that require comparatively small service times(multimedia applications), as long as their total service times cause insignificant delays to other packets in the queue. The algorithm provides delay guarantees to applications that do not contribute to congestion because of their tiny packet sizes and low transmission rates. The packets are prioritized and in turn corresponding flows, according to their impact on total delay. (Georgiou et al., 2011) introduced the mechanism based on the identification of high bandwidth from the drop history of Random Early Detect (RED). Bodin et al. (2000) proposed Weighted RED that calculates a separate average length for the high priority packets preventing starvation of the low priority packets. These methods demand costly memory structures.
(Dag, 2007), introduced static priority with deadline consideration, where the packets are given priorities based on the data type with multimedia data having high priority. If a network node becomes congested ' $k$ ' packets of the queue are sorted according to their priorities with the highest priority packet at the head of the queue. A packet with no remaining deadline is dropped. But sorting creates more overhead and time consuming.

Dimitriou et al. (2010), introduced Sizeoriented Dropping Policy (SDP) which derives the dropping policy from RED. RED drops the packets on calculated probability. Maximum threshold defines the region where forced dropping occurs. Minimum threshold defines the region where forced dropping occurs (Floyd and Jacobson, 1993). SDP records the size of each incoming packet to calculate the average packet size which serves as a guideline to differentiate small and big packets. If the arriving packet exceeds the average size (Big packets) it is dropped with RED probability, otherwise (for multimedia data) new probability will be calculated based on the size of the packet. In SDP gateways small packets experience less dropping probability than big packets compared to RED.

\section{PROPOSED SYSTEM}

The pseudocode of the proposed system is as follows:

1. Calculate the optimal packet size(opt_pkt_size) for the given encoding rate. The multimedia packets are split into the size calculated as opt_pkt_size.

2. In each node

Calculate the travel time(travel_time) from current node to destination from the routing table.

for each packet arrival

if remaining_deadline $<$ travel_time then drop the packet

else

$$
\begin{aligned}
& \text { if pkt_size } \leq \text { opt_pkt_size } \\
& \text { drop_prob }=\text { RED_drop } \\
& \text { else } \\
& \text { end if }
\end{aligned}
$$

end if

end for

3. Drop the packets using modified RED.

\subsection{Estimation of Optimal Packet Size}

\subsubsection{Input Parameters}

Number of intermediate nodes 
Buffer size

Bandwidth

Encoding rate (Size of the frames/second in $\mathrm{Kb}$ ).

Percentage of other data

Encoding rate

\subsubsection{Fixing the Threshold Values}

Minimum threshold- $1 / 4 *$ buffer size.

Maximum threshold- $3 *$ Minimum threshold

\subsubsection{Optimal Packet Size}

The packet size for which maximum amount of input data reaches the destination in minimal time is taken as optimal packet size(opt_pkt_size). The optimal packet size is estimated using exhaustive search.

1. The optimal packet size (opt_pkt_size) is calculated by considering the following parameters:
a. Available channel rate
b. Percentage of other data
c. Encoding rate

Throughput(Th) of multimedia data is estimated as follows Equation (1):

$\mathrm{Th}=\frac{((\text { ch_rate }- \text { other_data }) / \mathrm{p}-\mathrm{h})(\mathrm{p})+\text { other_data }}{\mathrm{N}}$

$\begin{array}{ll}\mathrm{p} & =\text { Optimal fragment size in bytes } \\ \text { ch_rate } & =\text { Channel rate in kbps } \\ \text { oth_data } & =\text { Other data rate in kbps } \\ \text { enc_rate } & =\text { Encoding rate in kbps } \\ \mathrm{N} & =\text { enc_rate }+ \text { other_data(Total data) }\end{array}$

The optimal size is determined as the size for which maximum data reach the destination in minimum time. In Equation 1 channel rate available after occupied by other data is divided into equal size fragments of multimedia data. It is added with other data. Throughput is calculated as the ratio of total data sent at the source side to total data received at the destination side.

Number of fragments that will be discarded can be estimated initially as follows (i.e.,) the multimedia packets that can be accommodated in the available channel rate will be discarded. The number of fragments discarded is given in the following Equation (2):

Frag - discard $=\frac{\text { enc }- \text { rate }- \text { other }- \text { date }}{p}$

\subsection{Packet Drop Based on Deadline}

The proposed algorithm calculates the travel time to the destination with the routing table information. If the estimated travel time of a packet is less than the remaining deadline the packet is dropped. This is implemented in $1^{\text {st }}$ node as well as in all nodes. This gives way for more useful packets (Packets with remaining deadline).

\subsection{Packet Drop Using Modified RED}

Packet size is the most typical and easy to extract indicator of the type of application that created the packet (Dimitriou et al., 2010). Transmission delay of a packet is proportional to the size of the packet. Small packets are utilized by the applications that require faster delivery, limited packet losses. Each packet has a deadline associated with it and the multimedia packets have to reach the destination before the expiry of the deadline. In case of congestion the packets are dropped based on the packet size and deadline. Optimal size packets have less dropping probability thereby reducing the packet drops of multimedia data.

Minimum threshold- $1 / 4 *$ buffer size.

Maximum threshold-3* Minimum threshold

When the average queue size is less than the minimum threshold, no packets are dropped. When the average queue size is greater than the maximum threshold, every arriving packet is marked. If the queue size is between the minimum and maximum threshold the packets are dropped with modified RED probability.

\subsubsection{Modified RED Algorithm}

\subsubsection{Initialization}

$\operatorname{Avg}=0$

Count $=-1$

for each packet arrival

calculate the average queue size(avg)

if $\min _{\text {th }} \leq$ avg $<\max _{\text {th }}$

calculate probability $\mathrm{p}_{\mathrm{a}}$ Equation 3 and 4:

$$
\mathrm{p}_{\mathrm{a}}=\frac{\mathrm{p}_{\mathrm{b}}}{1-\operatorname{count} * \mathrm{p}_{\mathrm{b}}}
$$

Where:

$\mathrm{p}_{\mathrm{b}}=\max _{\mathrm{p}} \frac{\left(\operatorname{avg}-\min _{\mathrm{th}}\right)}{\left(\max _{\mathrm{th}}-\min _{\mathrm{th}}\right)}$ 

end if

Mark the arriving packet with probability $\mathrm{p}_{\mathrm{a}}$ end for

count $=0$

\subsubsection{Drop Function of RED}

$$
\mathrm{pb}=\left\{\begin{array}{cc}
\frac{\operatorname{avg}-\min _{\text {th }}}{\max _{\text {th }}-\min _{\text {th }}} \max _{\mathrm{p}} \text { if } \min _{\text {th }}<\operatorname{avg}<\max \\
0 & \text { if avg }<\min _{\text {th }} \\
1 & \text { if avg }>\max _{\text {th }}
\end{array}\right.
$$

\subsubsection{Drop Function of Modified RED}

$$
\mathrm{p}_{\mathrm{b}}=\left\{\begin{array}{cc}
\frac{\mathrm{avg}-\min _{\mathrm{th}}}{\max _{\mathrm{th}}-\min _{\mathrm{th}}} \max _{\mathrm{p}} \text { if } \min _{\text {th }}<\mathrm{avg}<\max \\
0 & \text { if } \operatorname{avg}<\min _{\text {th }} \\
1 & \text { if } \operatorname{avg}>\max _{\text {th }}^{\text {th }}
\end{array}\right.
$$

\subsubsection{Notations}

\subsubsection{Saved Variables}

$$
\begin{array}{ll}
\text { Avg } & =\text { Average queue size } \\
\text { Count } & =\text { Packets since last marked packet } \\
\text { opt_pkt_size }= & \text { Calculated optimal packet size for } \\
& \text { multimedia data. } \\
\text { pkt_size } & =\text { Size of the arriving packet. }
\end{array}
$$

\subsubsection{Fixed Parameters}

Minth $=$ Minimum threshold for queue

Maxth = Maximum threshold for queue

Maxp = Maximum value for $\mathrm{pb}$

\subsubsection{Other}

pa: Current packet-marking probability

\section{EXPERIMENTAL RESULTS}

Videos pre-encoded using H.264/AVC is used to adopt the coding structure of Group of Pictures (GOP). GOP structure specifies the order in which intra- and interframes are arranged. A GOP contains the following picture types: I frame, $\mathrm{P}$ frame and $\mathrm{B}$ frame. Every GOP starts with an I-frame followed by a series of Pframe and B-frame. The typical Group of Pictures
(GOP) structure is IBBPBBP... I-picture or I-frame (intra coded picture)-reference picture, which represents a fixed image and which is independent of other picture types. P-picture or P-frame (predictive coded picture) - contains motion-compensated difference information from the preceding I- or Pframe. B-picture or B-frame (bidirectionally predictive coded picture) - contains difference information from the preceding and following I- or Pframe within a GOP (Peng and Zhang, 2010).

According to the inter frame dependency relationships it can be derived that $B$-frame could be dropped at any time. When a P-frame is dropped all other P- and B-frames following this frame should be dropped. When an I-frame is dropped that all other Pand B-frames following within the same GOP should be dropped.Hence I-frame has the highest priority followed by P-frame and B-frame.

The simulation is performed using NS2. Distance vector routing algorithm is used where the vector contains distances to all nodes in the network so that the packet's travel time could be calculated tentatively.

The video is assumed to be encoded in H.264/AVC and the frame size is taken as $16 \mathrm{~KB}$. Each frame has $\mathrm{I}, \mathrm{B}$ and $\mathrm{P}$ type frames.

\subsection{Steps}

1. Search the 2 consecutive I frames and estimate the play window by subtracting the time stamp values of the I-frames of two consecutive GOPs for given frame rate.

2. Fragment the packets into optimal size where each types of frame (I,B,P) is grouped as different set of fragments

3. Assign weight to each fragment based on priority. Iframe has the priority 3, P-frame has the priority-2 and B-frame has the priority-Weight of each fragment is priority*(Travel_time/deadline) which will change dynamically during travel

4. Mix with $50 \%$ of other data

5. Sort the fragments according to weight within each GOP with other data at the end of each GOP.

The structure of GOP is IPBBPBBPBBPBB. A low motion clip is assumed where I frame constitutes $50 \%$, P frames $25 \%$ and B frames occupy $25 \%$ of the total frame space

6. In each intermediate node check if entire GOP could be sent before deadline send the GOP without dropping any fragment else if I fragments and P-fragments could be sent drop B frames

else if I-fragments could alone be sent 
drop P frames and all B and P frames following it in the GOP

else

drop the entire GOP

(Travel time is calculated from the distance vector routing table).

7. In all the cases of step 6 send other data following each GOP without dropping.

8. Continue step 2-7 for different fragment sizes for the given frame rate.

9. Continue steps 1-8 for different frame rates given in Table 1.

10. Calculate the weighted throughput multiplying weight of fragment type (I,B,P) with the number of fragments of each type received.

Table 1 shows the determined optimal packet size for different encoding rate with channel rate $2 \mathrm{Mbps}$ and other data constituting $50 \%$ of the original data.

The simulation result for throughput for different encoding rates is given as graph in Fig.1. Channel rate is assumed to be 2 Mbps for different encoding rates. Figure 2 compares the throughput of SDP and modified RED for some encoding rates.

It is observed from Fig. 1 that maximum throughput is gained with optimal fragment size for each encoding rate. For example for encoding rate $640 \mathrm{kbps}$ maximum throughput is achieved with fragment sizes ranging from 250 to 480 bytes and for $896 \mathrm{kbps} 200$ to 400 bytes. But the maximum fragment size is used to avoid overhead. But for higher encoding rates the throughput is observed to be low because the data rate exceeds the channel rate leading to more fragment drops. When fragment size becomes high the throughput decreases proportionally because of the excess padding of data in each fragment. For example if the data to be fragmented is of size 1700 bytes. If the fragment size is 300 bytes the data may be split into 6 fragments with the 6th fragment padded with 100 bytes. But if the fragment size is 1500 bytes the data is split into 2 fragments with 1300 bytes padded in the 2nd fragment. With fragment size 300 bytes only 100 bytes extra has to be transmitted but in 1500 bytes fragment size 1300 bytes of extra data has to be sent.

The simulation results for packet drop using modified RED is given graphically in Fig. 2.

It is observed from Fig. 2 that packet drop was less in modified RED than in SDP.

The throughput is affected by the proportion of other data added with video data. The simulation result is shown as graph in Fig. 3.

From Fig. 3 it is observed that when more other data is added the data rate reaches the channel rate leading to more fragment drops due to deadline violations.

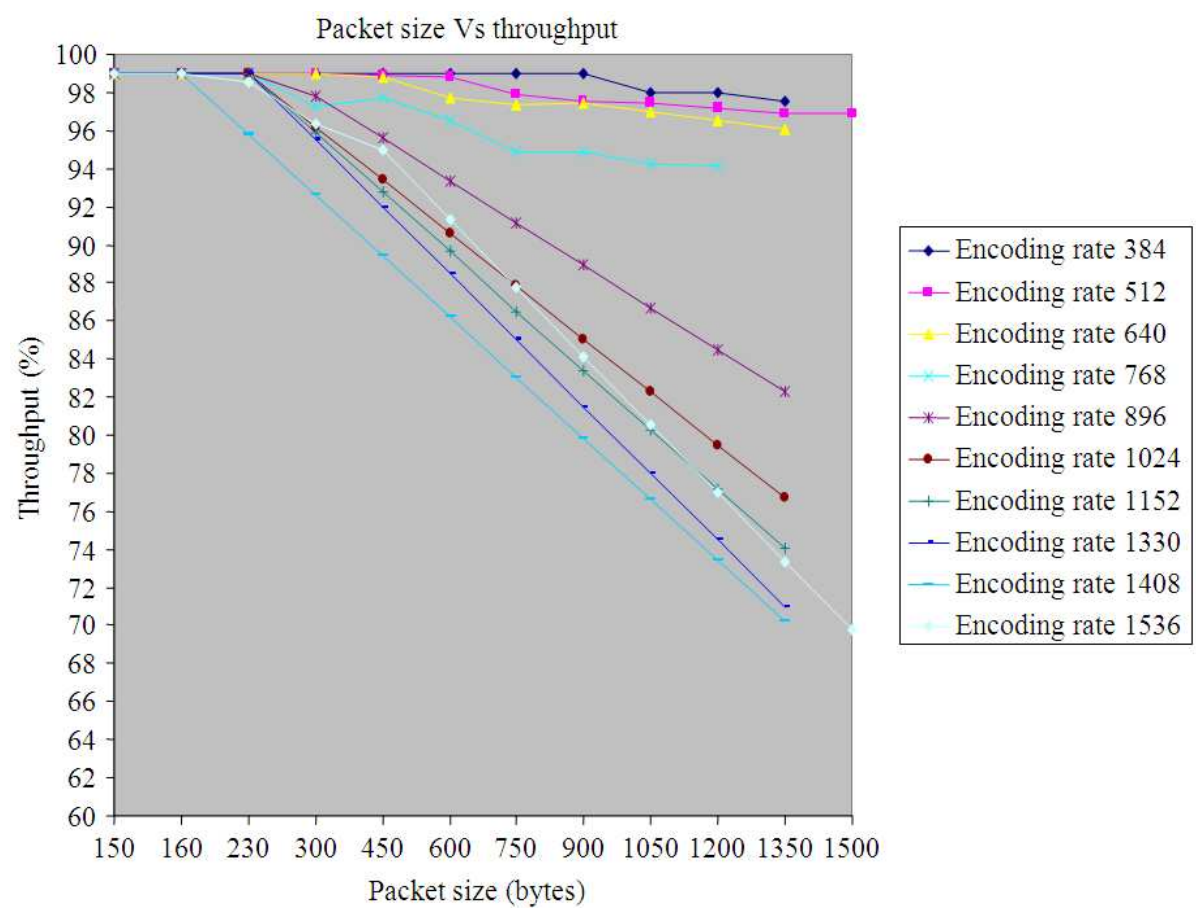

Fig. 1. Packet size vs throughput for different encoding rates 
K. Sasi Kala Rani and J. Suganthi / Journal of Computer Science 10 (7): 1336-1343, 2014

Table. 1. Optimal packet sizes for different encoding

\begin{tabular}{llcc}
\hline S. No & Frames/second & $\begin{array}{c}\text { Encoding rate } \\
\text { (in kbps) }\end{array}$ & $\begin{array}{c}\text { Optimal packet } \\
\text { size (in bytes) }\end{array}$ \\
\hline 1 & 3 & 384 & 680 \\
2 & 4 & 512 & 582 \\
3 & 5 & 640 & 480 \\
4 & 6 & 768 & 482 \\
5 & 7 & 896 & 400 \\
6 & 8 & 1024 & 382 \\
7 & 9 & 1152 & 332 \\
8 & 10 & 1330 & 320 \\
9 & 11 & 1408 & 232 \\
10 & 12 & 1536 & 160 \\
\hline
\end{tabular}
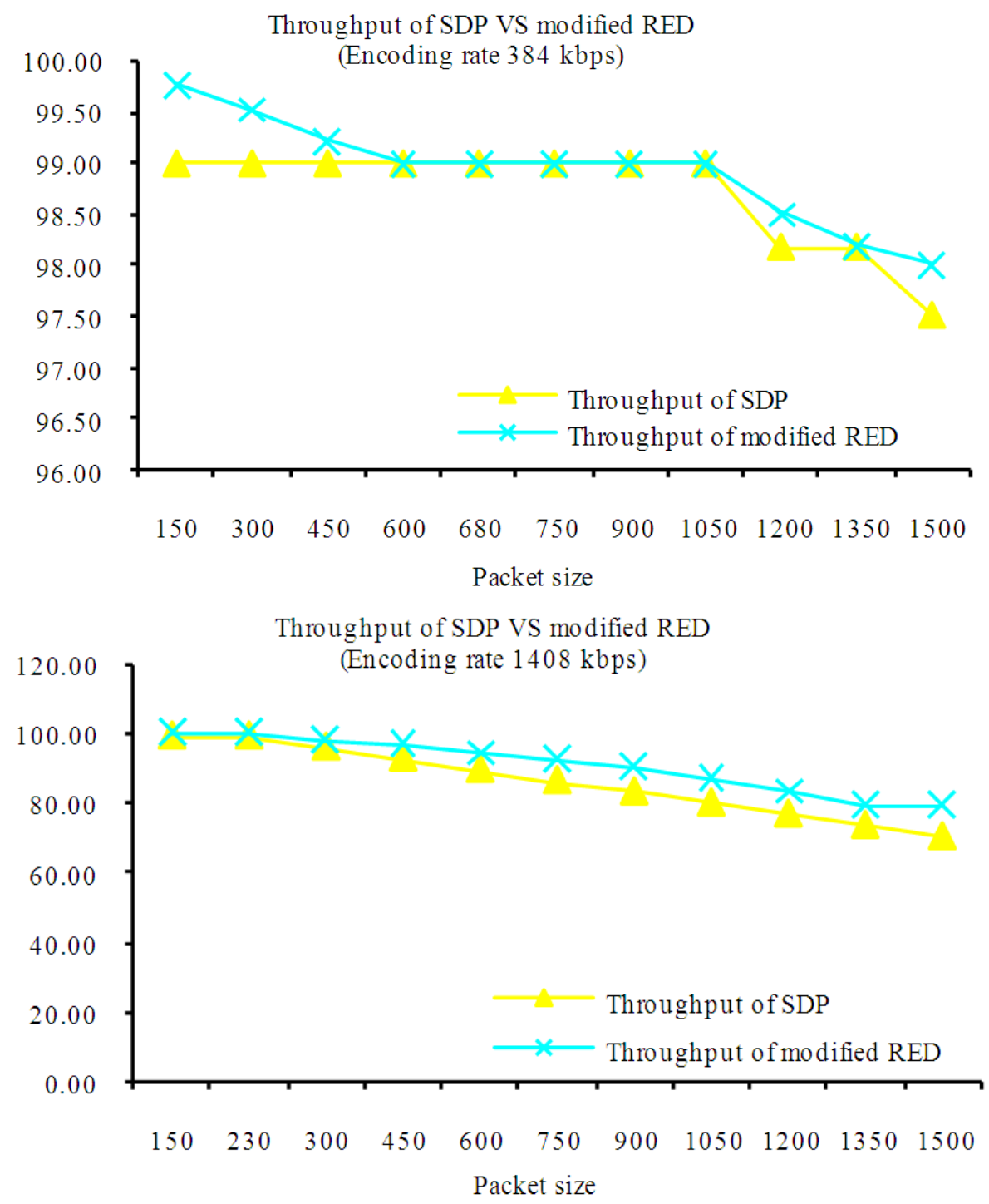

Fig. 2. Comparison of throughput of SDP and modified RED 


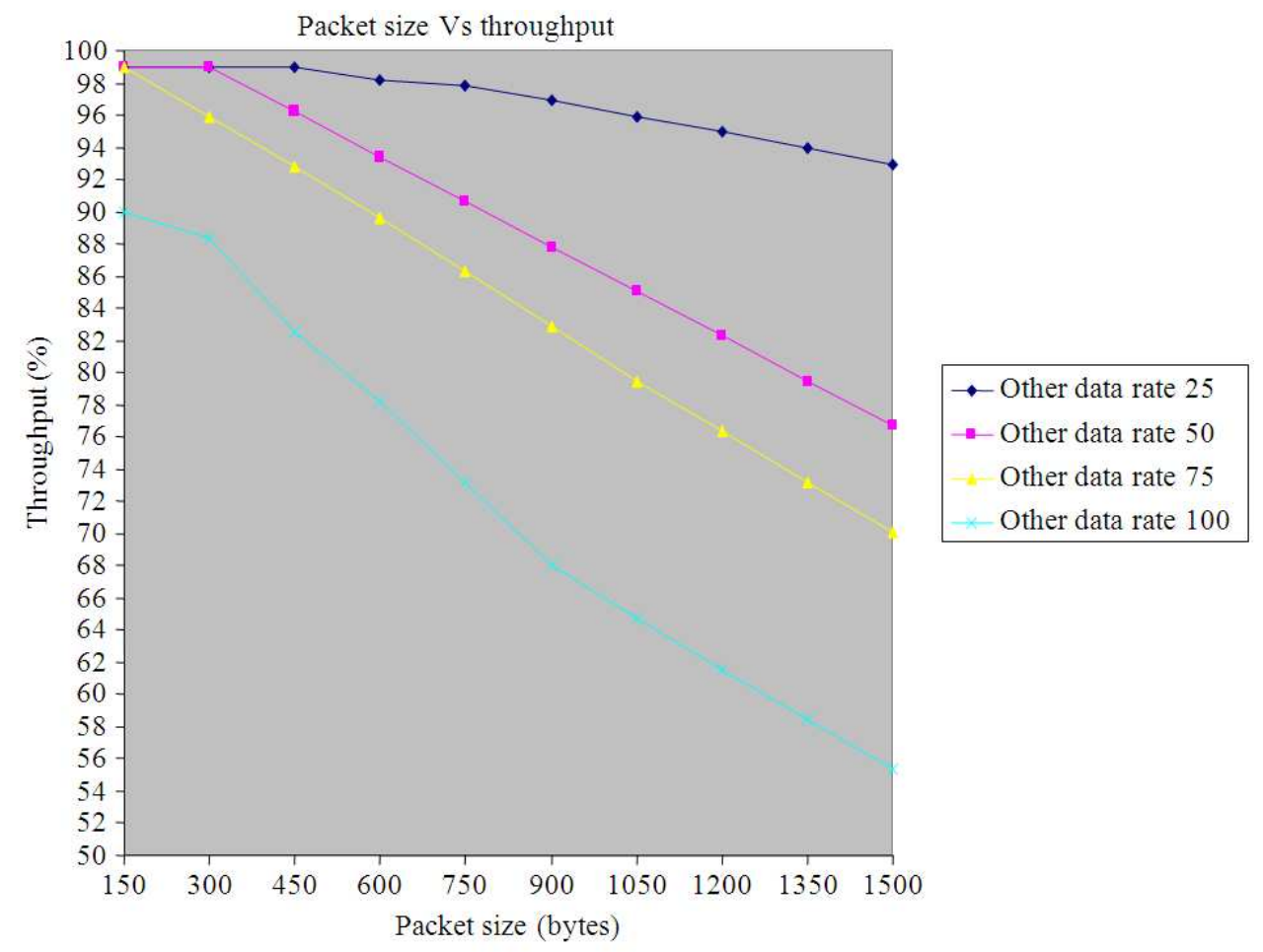

Fig. 3. Packet size vs throughput for different other data rates

\section{SUMMARY AND CONCLUDING REMARKS}

The proposed optimal fragmentation and modified RED algorithms are used to improve the QoS service of multimedia applications by improving the throughput. The packets are dropped based on the remaining deadline of the packet and packet size. The experimental evaluation shows

i. Efficient link utilization

ii. Increase in the arrival of useful packets to the destination thereby increasing the perceived quality of multimedia data.

iii. The fairness of other packets (non multimedia) are not affected..

The effect of delay and jitter on the perceived quality can also be studied.

\section{REFERENCES}

Bodin, U., O. Schelen and S. Pink, 2000. Load-tolerant differentiation with active queue management. ACM SIGCOMM Comput. Commun., 30: 4-160. DOI: $10.1145 / 382179.382180$
Bourenene, M., A. Mellouk and D. Benhamamouche, 2008. Inductive Qos packet scheduling for adaptive dynamic networks. Proceedings of IEEE International Conference on Communications, May 19-23, IEEE Xplor Press, Beijing, pp: 3090-3094. DOI: 10.1109/ICC.2008.581

Dag, T., 2007. SPD (Static Priority with Deadline Considerations) Packet Scheduling Algorithm for achieving better QoS. Proceedings of 3rd International Conference on Networking and Services, Jun. 19-25, IEEE Xplore Press, Athens, pp: 57-57. DOI: 10.1109/ICNS.2007.111

Dimitriou, S., A. Tsioliaridou and V. Tsaoussidis, 2010. Introducing size-oriented dropping policies as Qossupportive functions. IEEE Trans. Netw. Service Manage., 7: 14-27.

Floyd, S. and V. Jacobson, 1993. Random early detection gateways for congestion avoidance. IEEE/ACM Trans. Network., 1: 397-413. DOI: 10.1109/90.251892

Georgiou, C. and L. Mamatas, G. Papastergiou and V. Tsaoussidis, 2011. On short packets first: A delay oriented prioritization policy. Int. J. Commun. Syst., 24: 1065-1086. DOI: 10.1002/dac.1215 
Kambhatla, K.K.R., S. Kumar, S. Paluri and P.C. Cosman, 2012. Wireless H.264 Video quality enhancement through optimal prioritized packet fragmentation. IEEE Trans. Multimedia, 14: 1480-1495. DOI: 10.1109/TMM.2012.2196508

Li, F., 2009. Competitive scheduling of packets with hard deadlines in a finite capacity queue. Proceedings of the IEEE INFOCOM Infocommm, Apr. 19-25, IEEE Xplore Press, Rio de Janeiro, pp: 1062-1070. DOI: 10.1109/INFCOM.2009.5062018
Mamatas, L. and V. Tsaoussidis, 2010. Less Impact Better Service (LIBS). Annals Telecommun. Annales des Telecommun., 65: 447-459. DOI: 10.1007/s12243-010-0175-1

Peng, X. and X. Zhang, 2010. An unequal packet loss protection scheme for H.264/AVC video transmission. Proceedings of International Conference on Information Networking, Jan. 21-24, IEEE Xplore Press, Chiang Mai, pp: 1-5.

Steinmetz, R. and K. Nahrstedt, 2004. Multimedia Systems, 1st Edn., Springer, ISBN-10: 3540408673 